\title{
Low Frequency Signal Injection for Grid Impedance Estimation in Three Phase Systems
}

\author{
Pablo García, Juan M. Guerrero, Jorge García, \\ Ángel Navarro-Rodríguez \\ Dept.of Elec., Computer \& System Engineering \\ University of Oviedo \\ Gijón, 33204, Spain \\ Email: garciafpablo@uniovi.es, guerrero@uniovi.es, \\ garciajorge@uniovi.es, angelnr23@gmail.com
}

\author{
Mark Sumner \\ Department of Electrical and Electronic Engineering \\ The University of Nottingham \\ Nottingham, NG7 2RD, UK \\ Email: Mark.Sumner@nottingham.ac.uk
}

\begin{abstract}
This paper deals with the estimation and decoupling of grid impedance and LCL filter parameters variation using signal injection techniques and Luenberger type observer. When integrating a power converter in the $\mathrm{AC}$ grid as an interface for any Distributed Generation Systems (DGS) or other grid quality compensator like Active Power Filters (APF) or STATCOM, inner control loop normally requires current control. Current controller performance is greatly affected by the filter and grid impedance values. Although normally the filter impedance dominates the dynamics of the current controller, in weak networks the impedance of the grid can not be neglected. Additionally, other often required functions, as islanding detection, also rely on the estimation of the grid impedance. For this paper, a Luenberger based observer is proposed for controlling the grid current when a LCL filter is used. The proposed method will rely on measuring the converter side current and the grid voltage and will cope with parameter variation at the filter transfer function. For variations at the grid impedance, the control action deliver by the observer feedback path will be used for triggering an injection mechanism. A Low Frequency Signal Injection (LFSI) approach is proposed for online estimating the grid impedance using an RLS algorithm. The proposed estimation technique is well suited to be incorporated into an adaptive current controller scheme.
\end{abstract}

\section{INTRODUCTION}

Use of Voltage Source Converters (VSC) interfaced to the $\mathrm{AC}$ grid requires to control the current deliver to the grid. In order to accurately design the current controller, it is critical to understand the existing dynamic model between the applied converter output voltage and the resulting grid current. The dynamic model will affect in different ways the performance, depending on the used sensors and the filter used for the connection of the converter to the grid. When using a LCL filter for the interface, there exist multiple options for the placement of the current and voltage sensors, each of those with their advantages and drawbacks [1], [2]. When the control of the AC voltage at the output of the converter is needed, it is

This work has been partially funded by the Campus of International Excellence (CEI) of the University of Oviedo, Spain, in the framework of Mobility Grants for Academics in 2013. This work also was supported in part by the Research, Technological Development and Innovation Program Oriented to the Society Challenges of the Spanish Ministry of Economy and Competitiveness under grant ENE2013-44245-R and by the European Union through ERFD Structural Funds (FEDER). usual to measure the voltage at the filter capacitor. However, this will make the current controller dependent on the grid side impedance. The variation is effectively decoupled by moving the voltage sensor to the Point of Common Coupling (PCC), but then the capacitor voltage/current needs to be estimated in order to damp the current controller. The issue is more critical when interfacing the power converter to weak networks having a non negligible grid impedance, thus affecting the total output impedance. In order to overcome this problem there are two different alternatives: 1) to force the known output filter to be the dominant dynamic system in any grid situation by implementing passive/active damping or virtual impedance techniques [3], [4] and, 2) to implement and adaptive current controller [5], [6] which parameters change depending on the grid impedance.

For this second option, it is needed to online estimate the grid impedance and the variations at the LCL filter parameters. Impedance estimation could be implemented using two different approaches: 1) model based techniques and, 2) signal injection based techniques. Model based techniques, use the transfer function between the applied voltage and the current for estimating the parameters. In [7], the use of the resonance of a LCL filter is proposed in order to make the estimation. As commented by the authors, the principal issue of this technique is the existence of two resonance peaks when passive reactive power compensation is added at the Point of Common Coupling (PCC).

Signal injection based methods use an additional excitation in order to track the response of the system [8]-[11]. For the signal excitation, several approaches can be followed. 1) High Frequency Signal Injection (HFSI) [10]-[13], 2) current regulator reaction [11], 3) Low Frequency Signal Injection (LFSI) [14], [15]. 1) and 2) are based on the same physical explanation. When injecting a voltage at a given high frequency, the resulting current includes a component at that frequency. By analyzing those current components, it is possible to obtain the impedance. However, there are some issues with this high frequency injection techniques: 1) selection of the high frequency must be done by asserting that the reaction of any APF connected to the same PCC is not removing the high 


$$
\frac{d}{d t} \mathbf{x}=\left(\begin{array}{cccccc}
-R_{1}^{x} / L_{1}^{x} & \omega_{e} & -1 / L_{1}^{x} & 0 & 0 & 0 \\
-\omega_{e} & -R_{1}^{y} / L_{1}^{y} & 0 & -1 / L_{1}^{y} & 0 & 0 \\
1 / C & 0 & 0 & \omega_{e} & -1 / C & 0 \\
0 & 1 / C & -\omega_{e} & 0 & 0 & -1 / C \\
0 & 0 & 1 / L_{2}^{x} & 0 & -R_{2}^{x} / L_{2}^{x} & 0 \\
0 & 0 & 0 & 1 / L_{2}^{y} & 0 & -R_{2}^{y} / L_{2}^{y}
\end{array}\right) \cdot \mathbf{x}+\left(\begin{array}{cccc}
1 / L_{1}^{x} & 0 & 0 & 0 \\
0 & 1 / L_{1}^{y} & 0 & 0 \\
0 & 0 & 0 & 0 \\
0 & 0 & 0 & 0 \\
0 & 0 & -1 / L_{2}^{x} & 0 \\
0 & 0 & 0 & -1 / L_{2}^{y}
\end{array}\right) \cdot \mathbf{u}
$$

frequency current harmonic; 2) the estimated impedance is not the transient impedance, which is the one needed for tuning the current regulator, but the impedance at the injection frequency.

In order to overcome the aforementioned problems, in this paper a mixed strategy based on an observer and LFSI is proposed. By one side, a Luenberger style observer will be used for controlling the grid current with a reduced number of sensors. By the other, the proposed LFSI, consisting on the injection of a pulse aligned with the zero crossing of each three phase voltages, will allow to detect changes at the grid impedance. The pulse is open loop injected by modifying the voltage command delivered by the current controller. In order to reduce the disturbance in the grid, injection of the pulse is restricted to those time intervals in which a change in the grid impedance is detected by the observer. Errors in the feedback path of the observer are proposed to trigger the signal injection mechanism.

When compared with HFSI, the following differences are found: 1) the estimation of the grid transient impedance could be directly obtained, whereas when using the high frequency signal injection just the impedance at a given frequency is estimated; 2) the LFSI is more rich in harmonic content. This could help in reducing the impact of any APF connected in parallel with the converter injecting the low frequency signal; 3) signal processing is more complicated with the LFSI. In the case of HFSI, the estimation could be directly estimated from the isolated components at the injection frequency. Isolating the components just require the use of band-pass or low-pass filters in the stationary or carrier signal frequency respectively. For the LFSI, a model approach estimation based on Recursive Least Square (RLS) method is used. However, there is no need for any additional filter stage; 4) HFSI is affected by the transients in the fundamental current, being quite challenging to remove the transient harmonics using digital filters. In [16], the use of a fundamental current observer is proposed in order to mitigate the effect. On the contrary, using the proposed LFSI, fundamental transient currents could also be used for the impedance estimation.

The paper is organized at follows. Section II explains the state space model of the LCL filter and grid impedance, the design of the observer and the digital implementation of the control system. Section III shows the injection mechanism, including the selection of the injection pulse and Section IV the RLS adaptive procedure used for the grid impedance estimation. Finally, simulation and experimental results are shown at Section V.

\section{SYSTEM MODELING AND CONTROL}

This section describes the system modeling in a generic reference frame as well as the theoretical background for the implemented control. It also includes the details for the digital implementation of the designed Luenberger observer.

\section{A. System modeling}

The state space representation of a $L C L$ filter (Fig. 1) in an arbitrary reference frame is given by (1), where $\mathbf{x}=$ $\left[\mathbf{i}_{i}, \mathbf{v}_{c}, \mathbf{i}_{g}\right]^{T}$ is the state vector and $\mathbf{u}=\left[\mathbf{v}_{i}, \mathbf{v}_{g}\right]^{T}$ the input vector. Also note that each component at the state and input vectors is a complex variable with two elements; the real and imaginary components, i.e $\mathbf{v}=\left(v_{x}, v_{y}\right)$. Equation (1) could be particularized for the stationary $(\alpha, \beta)$ or to the synchronous $(d, q)$ references frames by making $\omega_{e}=0$ or $\omega_{e}=\omega_{\text {grid }}$ respectively. An alternative representation of (1), separating the $x$ and $y$ terms, is shown at (2), (3) and in compact form at (4), (5). That form will be used for an easier digital implementation of the observer structure. Finally, the corresponding block diagram representation in compact complex notation is shown at Fig. 2.

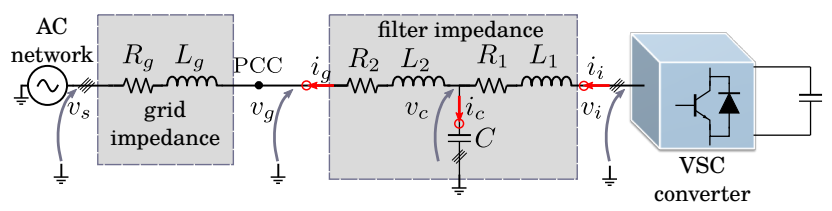

Fig. 1. Connection of the LCL filter to the output of the VSC converter.

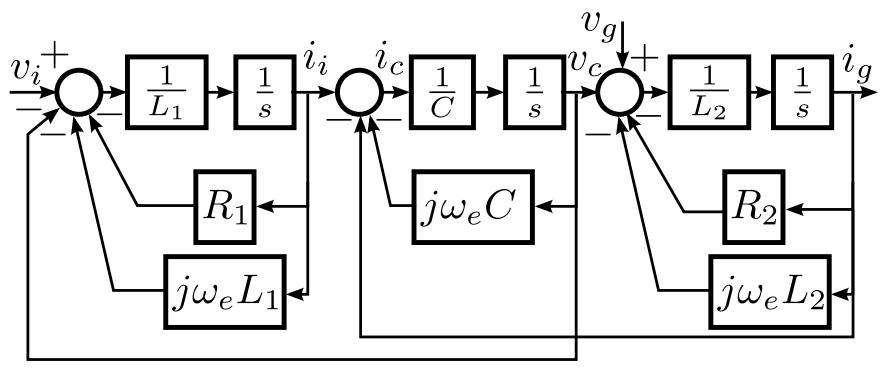

Fig. 2. LCL filter block diagram in state space form. 


$$
\begin{aligned}
\frac{d}{d t} \mathbf{x}_{x} & =\left(\begin{array}{ccc}
-R_{1} / L_{1} & -1 / L_{1} & 0 \\
1 / C & 0 & -1 / C \\
0 & 1 / L_{2} & -R_{2} / L_{2}
\end{array}\right)_{x} \cdot \mathbf{x}_{x} \\
& +\omega_{e}\left(\begin{array}{lll}
1 & 0 & 0 \\
0 & 1 & 0 \\
0 & 0 & 1
\end{array}\right) \cdot \mathbf{x}_{y}+\left(\begin{array}{cc}
1 / L_{1} & 0 \\
0 & 0 \\
0 & -1 / L_{2}
\end{array}\right)_{x} \cdot \mathbf{u}_{x}
\end{aligned}
$$$$
\begin{aligned}
\frac{d}{d t} \mathbf{x}_{y} & =\left(\begin{array}{ccc}
-R_{1} / L_{1} & -1 / L_{1} & 0 \\
1 / C & 0 & -1 / C \\
0 & 1 / L_{2} & -R_{2} / L_{2}
\end{array}\right)_{y} \cdot \mathbf{x}_{y} \\
& -\omega_{e}\left(\begin{array}{lll}
1 & 0 & 0 \\
0 & 1 & 0 \\
0 & 0 & 1
\end{array}\right) \cdot \mathbf{x}_{x}+\left(\begin{array}{cc}
1 / L_{1} & 0 \\
0 & 0 \\
0 & -1 / L_{2}
\end{array}\right)_{y} \cdot \mathbf{u}_{y}
\end{aligned}
$$

$$
\begin{aligned}
\frac{d}{d t} \mathbf{x}_{x} & =\mathbf{A}_{x} \cdot \mathbf{x}_{x}+\omega_{e} \mathbf{I} \cdot \mathbf{x}_{y}+\mathbf{B}_{x} \cdot \mathbf{u}_{x} \\
\frac{d}{d t} \mathbf{x}_{y} & =\mathbf{A}_{y} \cdot \mathbf{x}_{y}-\omega_{e} \mathbf{I} \cdot \mathbf{x}_{x}+\mathbf{B}_{y} \cdot \mathbf{u}_{y}
\end{aligned}
$$

\section{B. Control implementation}

The superior filtering performance of the LCL structure when compared to the $\mathrm{L}$ or $\mathrm{LC}$ alternatives has also important shortcomings in the design of the current controller [17]. This situation is even worsen when harmonic compensation is considered [18]. Current control for a LCL filter is a challenging task due to the resonance appearing between the capacitor and the inductances and normally an attenuation method is needed. The basic idea is to compensate or attenuate the capacitor current within the bandwidth of the current controller, but still keeping the filtering capability for frequencies at and above the switching frequency.

In the literature there are several alternatives which can be separated into passive and active damping techniques. By one side, passive damping techniques require the use of additional passive elements, such a series or parallel resistances which increase the system losses [17]. By the other, active damping methods often needs for additional current or voltage sensors. Lately, some publications have addressed the implementation of active damping methods which do not need any for extra elements [2], [19]-[24]. The methods in that group could be separated in those which require to estimate the capacitor current or the inductance voltage, thus relying on calculating derivatives which are normally noisy or require the use of complicated control algorithms. More appealing because of their simplicity are those methods relying on digital filtering of the control signal in order not to react at the resonance frequency. However, often the bandwidth of the current controller must be decreased.

For this paper, an structure based on a Luenberger type observer is proposed [25]. The proposed system will control the grid side current by using the converter side current sensors and the voltage sensors at the PCC as the solely sensing elements. This configuration has some advantages in terms of 1) costs, only the current sensors usually provided by the power stage need to be used; 2) safety, the current sensors on the converter side are also suitable for protection of the power stage; 3 ) performance, PCC voltage measurement allows for measuring and decoupling the effects of a varying grid impedance but also to real power factor measurement and; 4 ), reliability as a reduced number of sensors reduces the fault probability.

The proposed observer and current control block diagram are shown at Fig 3. The control system works as follows. The estimated values for the converter side inductance $\left(\widehat{L}_{1}\right)$, filter capacitor $(\widehat{C})$ and grid side inductance $\left(\widehat{L}_{2}\right)$ are used for building the dynamic model previously shown in (1). Inputs to the observer are the commanded voltage from the converter $\left(v_{i}^{f f}\right)$ and the measured converter side current $\left(i_{i}\right)$. The estimated capacitor current $\left(\hat{i}_{c}\right)$ is obtained from the difference of the measured converter side current and the estimated grid current $\left(\widehat{i}_{g}\right)$. $\widehat{i}_{c}$ is later used at the output of the current controller to implement the active damping mechanism and $\widehat{i}_{g}$ is used as the feedback signal for the current controller. The feedback path of the observer is generated from the estimation error of the converter side current $\left(e_{i}=i_{i}-\hat{i}_{i}\right)$. The error signal is the input to the observer controller $\left(C_{o}\right)$ which, depending on the reference frame of the implementation, will be a PI (synchronous reference frame) or a PR for the stationary reference frame. The output of the feedback path $\left(v_{i}^{f b}\right)$ is added to the feedforward value to the commanded voltage in order to compensate for any unknown in the system. The feedback voltage will also be used for triggering the low frequency pulse injection. Finally, for the current controller implementation $\left(C_{i}\right)$, PI or PR structures are used depending on the selected reference frame. The active damping term and the measured PCC voltage are after added in order to damp the oscillations and to effectively reject the effects on the grid current due to any change in the grid impedance.

\section{Digital control implementation}

For the online implementation, the Luenberger observer and the current controller designs must be translated to the digital domain. The Luenberger observer is discretized using the Euler approximation, as shown in (6), (7), where $[k]$ corresponds to the actual sample time, $[k-1]$ to the previous one and $T_{s}$ is the sample time. It is worth noting than even if here the matrix formulation is shown for the sake of simplicity, the discrete observer equations can be implemented in scalar form, more suitable for the online implementation.

$$
\begin{aligned}
\mathbf{x}_{x}[k] & =\left(\mathbf{I}-T_{s} \mathbf{A}_{x}\right)^{-1} \cdot\left(\mathbf{x}_{x}[k-1]+T_{s} \mathbf{B}_{x} \cdot \mathbf{u}_{x}[k]\right) \\
& +T_{s} \omega_{e} \mathbf{I} \cdot \mathbf{x}_{y}[k-1] \\
\mathbf{x}_{y}[k] & =\left(\mathbf{I}-T_{s} \mathbf{A}_{y}\right)^{-1} \cdot\left(\mathbf{x}_{y}[k-1]+T_{s} \mathbf{B}_{y} \cdot \mathbf{u}_{y}[k]\right) \\
& -T_{s} \omega_{e} \mathbf{I} \cdot \mathbf{x}_{x}[k-1]
\end{aligned}
$$

For the controller discretization, Tustin approximation is used. PI or PR structures are used depending on performing the implementation at the synchronous or the stationary reference frame respectively. 


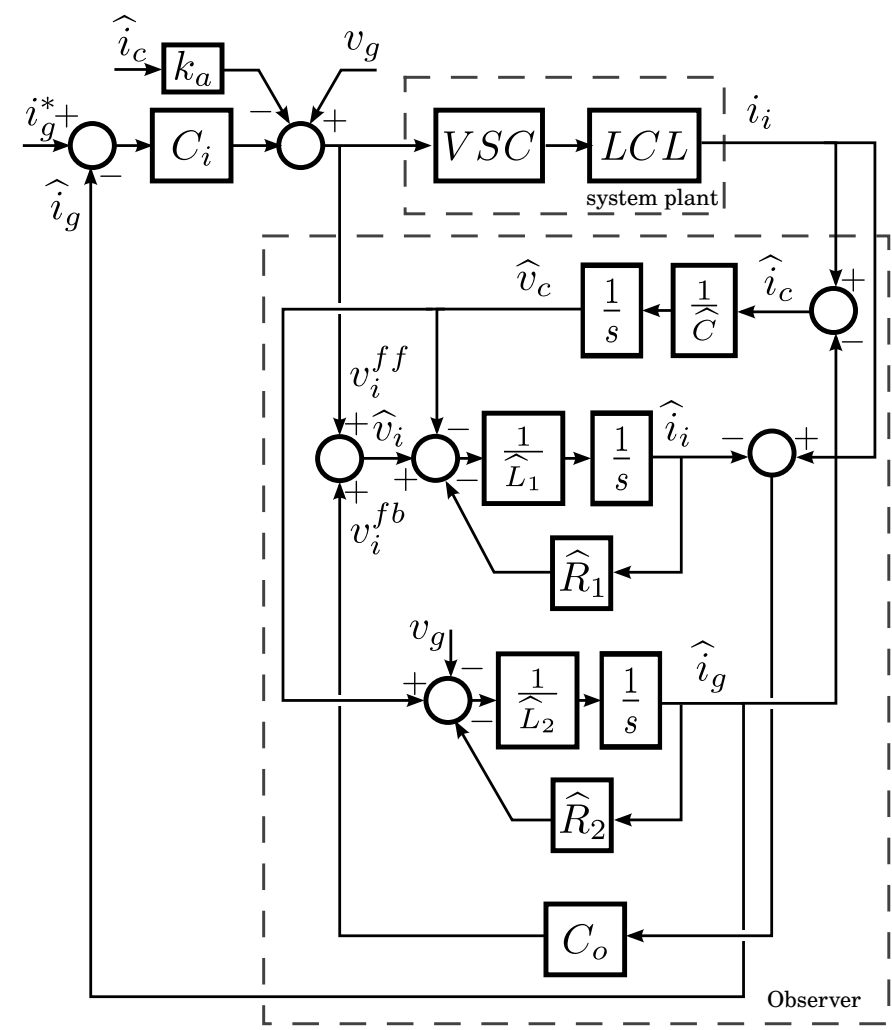

Fig. 3. Proposed observer structure in an arbitrary reference frame.

The observer performance, at the synchronous reference frame, is shown at Fig. 4 when the estimated LCL filter parameters match the real ones. As shown, the grid current is correctly tracked.

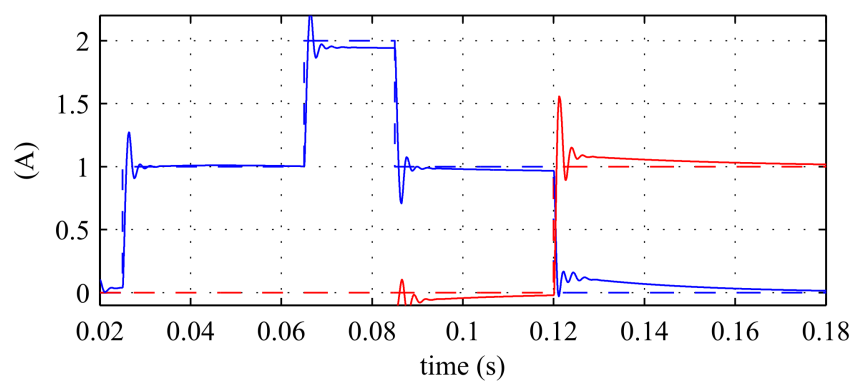

Fig. 4. Transient response for the presented observer structure in digital form.

\section{LOW FREQUENCY SIGNAL INJECTION}

For the LFSI, there are different alternatives and parameters which can be adjusted. As represented in Fig. 5, the signal is injected centered at the zero crossing of the phase to neutral voltages. Zero crossing is detected by using the calculated phase from the PLL used for grid synchronization. This point has been selected in order to minimize the voltage distortion, as demonstrated later in the discussion.

At this paper, three different alternatives for the pulse injection are investigated, the first two are implemented at the $a b c$ reference frame, while the third one is at the $d q$ reference frame. The pulses are injected as a duty modifications to the output of the current controller and, during the pulse injection, the fundamental voltage command is disabled for the case of the $a b c$ injection (see Fig. 6) whereas is just an addition when implemented in the $d q$ reference frame. This will enable both a sharper excitation but also a quite simple implementation of the signal injection. As seen in Fig. 5, both the pulse width and the magnitude can be changed. As wider is the pulse and as larger the magnitude, the bigger the disturbance delivered to the system would be. Obviously, increased disturbance values will help in the estimation procedure, but also will increase the THD of the resulting currents. For this paper, the values shown in Table I have been used. Resulting waveforms for the inverter commands and the applied voltages are shown at Fig. 7 whereas the corresponding currents at the synchronous reference frame are shown at Fig. 8. The three tested alternatives are following described:

1) Method\#1. Pulse width is established to the desired value and the the magnitude is set to zero. Under that condition, the fundamental voltage command is clamped to zero during the pulse injection time. The implementation of this strategy is straightforward, since it is just a multiplication of the duty commands times a time window set to zero during the pulse duration. When translating to the $d q$ reference frame, even if the pulse is mostly at the $q$ axis, both components are modified. The pulses are transformed to a triangular shape at the $q$ axis and the resulting current has a sinusoidal waveform.

2) Method\#2. Fundamental command is hold at the corresponding value at the beginning of the pulse injection and when the phase crosses the zero is changed to the opposite value. Transformed to the $d q$ reference frame, $d$ component is also modified, although in a less noticeable way than for Method\#1. The pulses at the $q$ axis are also transformed to a triangular shape, but the resulting current has a triangular waveform of opposite phase when compared to previous method.

3) Method\#3. When looking at the pulse result in the $d q$ reference frame for both Method\#1 and Method\#2, the resulting excitation is affecting the $d$ and $q$ axis and, even if the pulse is an stepwise in the $a b c$ reference frame, is having a triangular form on the $d q$ reference frame. Because the RLS algorithm will be implemented in the synchronous reference frame, it is desirable to have an step shape in that reference. This could be easily achieved by using the same strategy than for Method\#2 but injecting the pulses directly in the synchronous reference frame at the $q$ axis.

It must be remarked that all pulse injection strategies share the fact that the applied distortion to the voltage command is symmetrical, thus resulting in the voltage average error being zero. Selecting one or the other is based on the sensitivity of the current response and on the implementation burden. For this paper, Method\#3 is considered because of the advantages 
enumerated before.

Previous simulation results were showing the injection mechanism with the system in open loop, however the power converter will operate under current control and the pulses injection will be seen as a disturbance for the current controller. In the case the current regulator reaction is too fast, the pulses will be removed from the excitation and the estimation could not be implemented. Experimental results of the system operating under close loop with a $200 \mathrm{~Hz}$ bandwidth are shown at Fig. 9. It is clear that even under close loop operation the pulses appear on the grid voltage and thus could potentially be used for the RLS estimation.

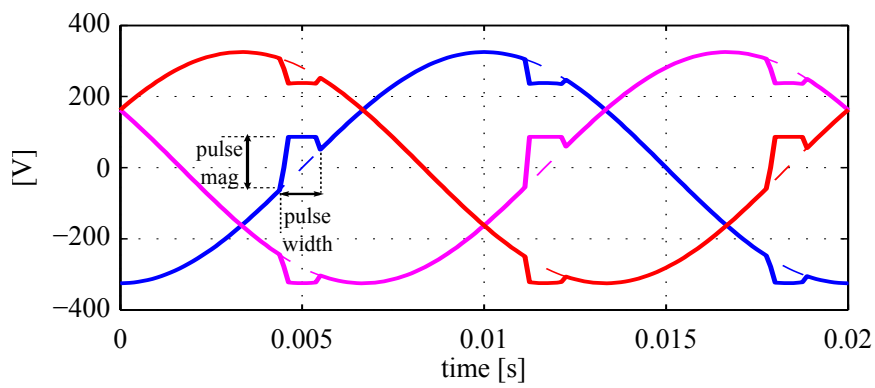

Fig. 5. LFSI pulse generation. Both magnitude and phase can be independently changed. The magnitude has been deliberately increased for illustration purposes.

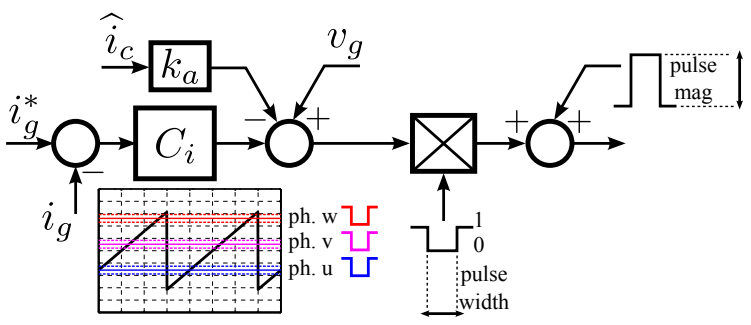

Fig. 6. LFSI implementation. The injection is synchronized and centered with respect to the grid voltage zero crossing and the fundamental voltage command is blanked during the injection time. Dashed lines show the starting and end of each phase pulse and bold ones the zero crossing of the respective phase.

\section{RLS ALGORITHM IMPLEMENTATION}

In order to obtain the grid impedance parameters, an estimation procedure needs to be implemented. In the literature there are mainly two approaches. To calculate the impedance as the quotient of the injected voltage and the resulting current [10] or to use an observer or an estimator [13]. In this paper, the estimation of the system parameters is done by using a RLS approach [26]. For that, the dynamic equation of the grid impedance in the synchronous reference frame as seen from the converter is first discretized using Euler method.

The matrix equation of a three phase $R L$ load in the synchronous reference frame, after decoupling the cross coupling terms, is given by (8). Where $\mathbf{v}_{R L}$ is the vector voltage drop across the impedance, $\mathbf{v}_{g}$ the PCC voltage vector, $\mathbf{v}_{s}$ the grid voltage vector, and $\mathbf{i}_{g}$ the grid current vector. $\mathbf{L}$ and $\mathbf{R}$ are, respectively, the inductance and resistance matrices.
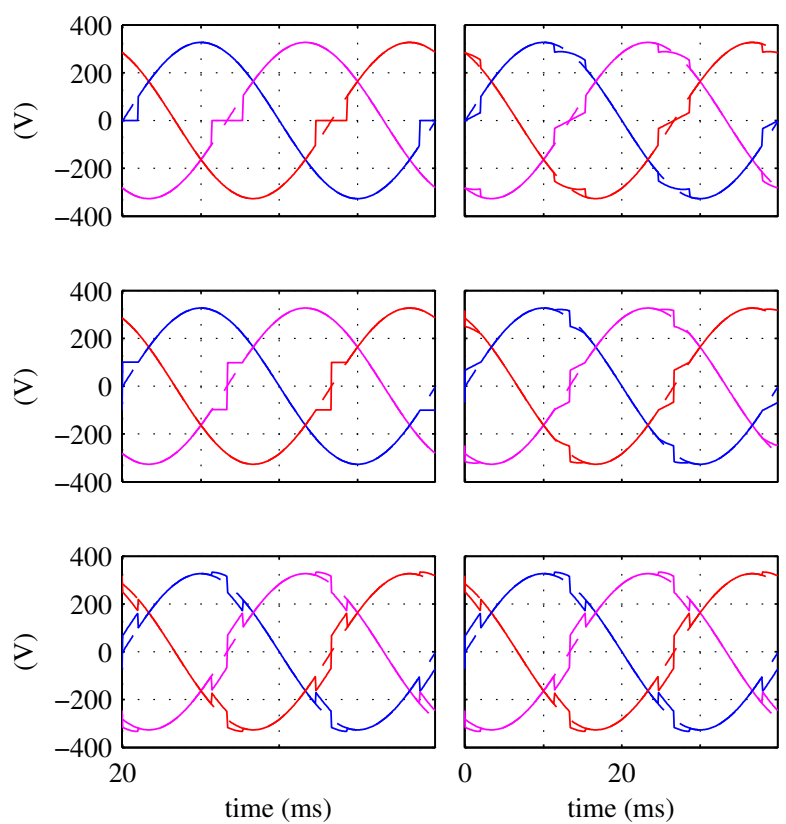

Fig. 7. LFSI waveforms for the three proposed methods in the $a b c$ reference frame. From top to bottom, Method\#1, Method\#2 and Method\#3. Left column shows the generated phase voltage command and right column the phase to neutral voltages. Dashed lines show the signals when the pulse injection is disabled.

$$
\mathbf{v}_{R L}=\mathbf{v}_{g}-\mathbf{v}_{s}=\mathbf{R} \mathbf{i}_{g}+\mathbf{L} \frac{d \mathbf{i}_{g}}{d t}
$$

When calculating the discrete approximation using Euler method with a sampling period $T_{s}$, equation (9) is obtained.

$$
\mathbf{i}_{g}[k]=\mathbf{a}_{\mathbf{1}} \mathbf{i}_{g}[k-1]+\mathbf{b}_{\mathbf{0}}\left(\mathbf{v}_{c}[k]-\mathbf{v}_{g}[k]\right)
$$

Where $\mathbf{a}_{1}$ and $\mathbf{b}_{\mathbf{0}}$ are given by expression (10)

$$
\mathbf{a}_{\mathbf{1}}=\frac{\mathbf{L}}{\mathbf{R} T_{s}+\mathbf{L}}, \quad \mathbf{b}_{\mathbf{0}}=\frac{T_{s}}{\mathbf{R} T_{s}+\mathbf{L}}
$$

From (10), the values for the resistance and inductance matrices can be obtained as (11) respectively.

$$
\mathbf{R}=\frac{1-\mathbf{a}_{\mathbf{1}}}{\mathbf{b}_{\mathbf{0}}}, \quad \mathbf{L}=\frac{\mathbf{a}_{\mathbf{1}} T_{s}}{\mathbf{b}_{\mathbf{0}}}
$$

It is worth noting that the above proposed model is valid for any balanced or unbalanced RL load. When the load is balanced, $\mathbf{a}_{1}=\left[a_{1 d}, a_{1 q}\right]$ components and $\mathbf{b}_{0}=\left[b_{0 d}, b_{0 q}\right]$ components will have the same value. For unbalanced loads, the resulting components will be different.

For the RLS implementation, the error between the measured and estimated current (12) is used to update the estimation. In order to decouple the effect of the unknown grid voltage, $v_{s}$, only the current generated by the pulses is used. This is done by subtracting from the overall current the reference of the fundamental current. It then assumed that the grid voltage at the pulse frequency is zero and 
TABLE I
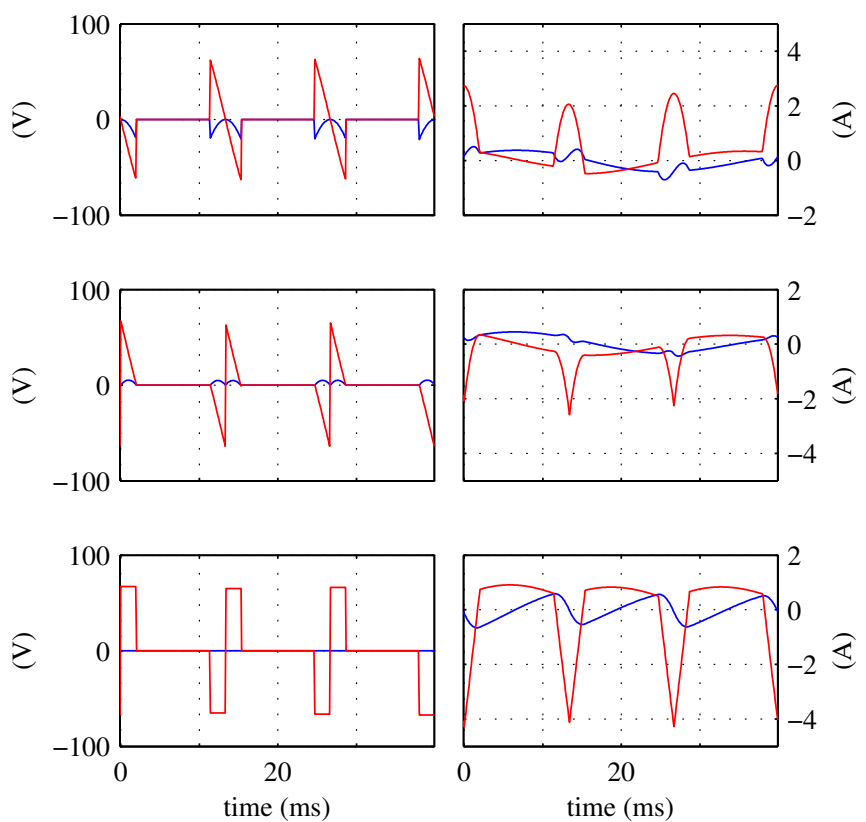

Fig. 8. LFSI waveforms for the three proposed methods in the $d q$ reference frame. Fundamental component is removed in order to zoom on the high frequency components. From top to bottom, Method\#1, Method\#2 and Method\#3.
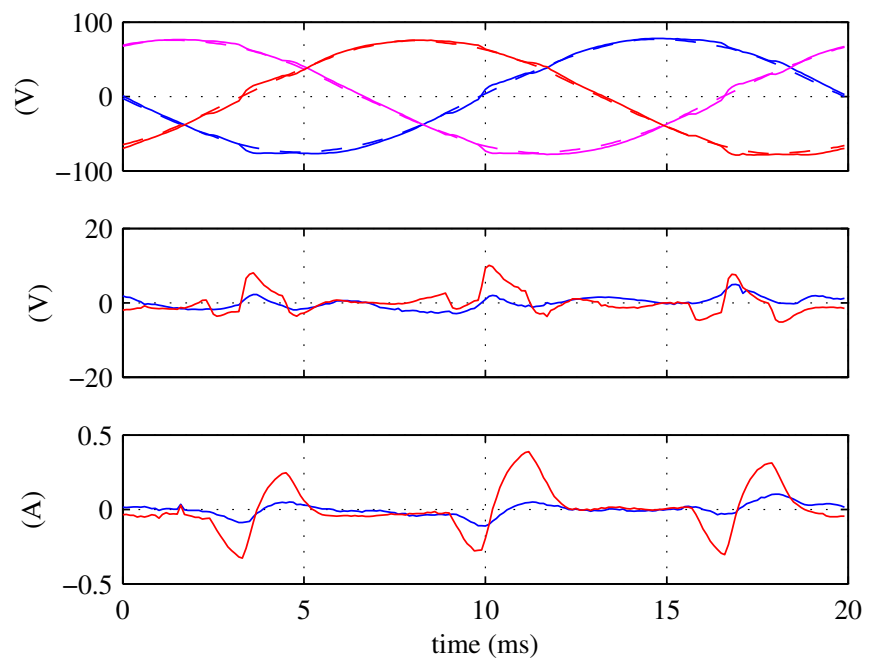

Fig. 9. Experimental results for Method\#3. The system is operated in close loop with a bandwidth of $200 \mathrm{~Hz}$. Filter capacitor is removed and current commands were set to zero. From top to bottom it is shown the phase to neutral voltages at the PCC, the voltage commands, and the grid currents at the synchronous reference frame. Blue color is used for the $d$ component and red for the $q$.

thus could be removed from the estimation. The system equations are represented in state-space form by defining the state vector $\mathbf{X}_{[k]}=\left[\mathbf{i}_{g_{[k-1]}}, \mathbf{v}_{\left.g_{[k]}\right]}\right]^{T}$ and coefficients vector $\mathbf{W}_{[k]}=\left[\mathbf{a}_{\mathbf{1}[k]}, \mathbf{b}_{\mathbf{0}[k]}\right]$
PARAMETERS

\begin{tabular}{c|c}
\hline Nominal parameters & Value (Setup\#1/Setup\#2) \\
\hline$r_{1}[\Omega]$ & $0.2 / 0.2$ \\
$r_{2}[\Omega]$ & $0.2 / 0.2$ \\
$L_{1}[m H]$ & $2.3 / 7$ \\
$L_{2}[m H]$ & $0.93 / 7$ \\
$C[\mu F]$ & $10 / 6$ \\
pulse mag. $[p . u]$ & $0.1 / 0$ \\
pulse width. $[m s]$ & $1 / 2$ \\
$\lambda$ & $0.998 / 0.8$ \\
\hline
\end{tabular}

$$
\mathbf{e}_{[k]}=\mathbf{i}_{g[k]}-\widehat{\mathbf{i}}_{g[k]}
$$

The least square problem is formulated in recursive form using the equations (13) - (16). $P_{(2 x 2)}$ is the covariance matrix and it is initialized to $P=0.01\left(\begin{array}{ll}1 & 0 \\ 0 & 1\end{array}\right), g_{(2 x 1)}$ the adaptation gain and $\lambda=[0,1]$ is the forgetting factor, which need to be selected as a tradeoff of the expected estimation bandwidth and the signal to noise ratio. Frequently, values between 0.95 and 1 are selected. For this paper, the value shown in Table I has been selected. At each sample time, the estimation of the parameters $\mathbf{b}_{\mathbf{0}}, \mathbf{a}_{\mathbf{1}}$ is updated and a new estimation for $\mathbf{R}$ and $\mathbf{L}$ is obtained.

$$
\begin{aligned}
\alpha_{[k]} & =\mathbf{i}_{[k]}-\mathbf{W}_{[k-1]} \cdot \mathbf{X}_{[k]} \\
g_{[k]} & =P_{[k-1]} \cdot \mathbf{X}_{[k]} \cdot\left[\lambda+\mathbf{X}_{[k]}^{T} \cdot P_{[k-1]} \cdot \mathbf{X}_{[k]}\right]^{-1} \\
P_{[k]} & =\lambda^{-1} \cdot P_{[k-1]}-g_{[k]} \cdot \mathbf{X}_{[k]}^{T} \lambda^{-1} \cdot P_{[k-1]} \\
\mathbf{W}_{[k]} & =\mathbf{W}_{[k-1]}+\left(\alpha_{[k]} \cdot g_{[k]}\right)^{T}
\end{aligned}
$$

\section{Simulation And ExPerimental Results}

For the simulation results, Simulink has been used for the implementation and Matlab for the analysis. All the simulation results were obtained using real time signal processing. The parameters' estimation and adaptation is continuously calculated at each simulation step. Fig. 10 shows a transient in the grid inductance from $7 \mathrm{mH}$ to $14 \mathrm{mH}$ and on the grid resistance from 0.2 to $0.25 \Omega$. The figure shows the estimation of the inductance and resistance for a balanced grid, the modified voltages with the pulse injection and the estimated and real currents. As it can be seen, the parameter estimation converges in a fraction of a fundamental cycle. It is also shown that the resistance estimation drops before reaching the final value. Explanation for that behavior is related with a peak in the estimation of the $a_{1}$ coefficient. Still the problem is not too important due to the high convergence speed.

Initial experimental results were obtained using a PM15F42C power module from Triphase. The power modules is directly programmed from Simulink environment, thus allowing for easily test the simulation results. The power module is interfaced to the AC grid trough a LCL filter, which parameters are listed in Table I under Setup\#1. In that table, the 

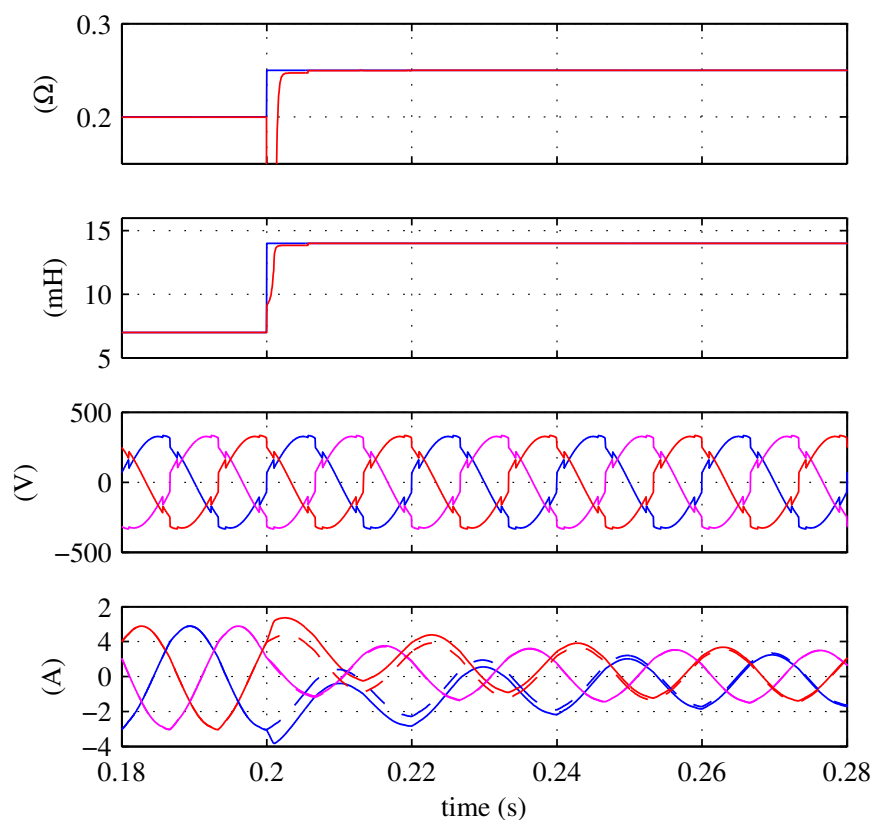

Fig. 10. Simulation results. Transient response. From top to bottom: a) resistance estimation, b) inductance estimation, c) applied voltages, d) resulting grid currents. Measured currents are shown in dashed lines, whereas estimated are in bold.

converter side inductance is $L_{1}$ and the grid side inductance $L_{2}$. An additional inductance of $L_{l}=5 \mathrm{mH}$ has been placed in series after $L_{2}$ in order to simulate a weak network. During these experiments, grid impedance estimation using Method\#1 were tested.

Fig. 11 shows the obtained waveforms and the corresponding currents during the experiments. By looking at the phase voltages it is clear that the pulse injection is working as expected.

Fig. 12 shows the estimated resistance and inductance during the experimental tests. By looking at the results, the inductance value is really well estimated (the sum of $L_{1}+L_{2}+L_{l}=8.4 \mathrm{mH}$ ). The value of the obtained resistance seems to be too high when compared to the measured one (around $0.5 \Omega$ ). A possible explanation could be the effect of the filter capacitance, which is reducing the amount of pulsating current reaching the grid side current sensor.

Additional experiments, including the online implementation of the observer, the development and testing of Method\#3 and the RLS implementation in the synchronous reference frame have been taken using a MTL-CBI0010N12IXFE power stage from Rectificadores Guash and the control being implemented in a TMS320F28335 DSC from Texas Instruments. The details about the grid filter and pulse injection parameters are shown at Table I under Setup\#2.

Experimental results for the the observer reference tracking capability are shown at Fig. 13. Fig. 14 shows the estimated and real currents using Method\#3 for the pulse injection and RLS at the synchronous reference frame at steady state con-
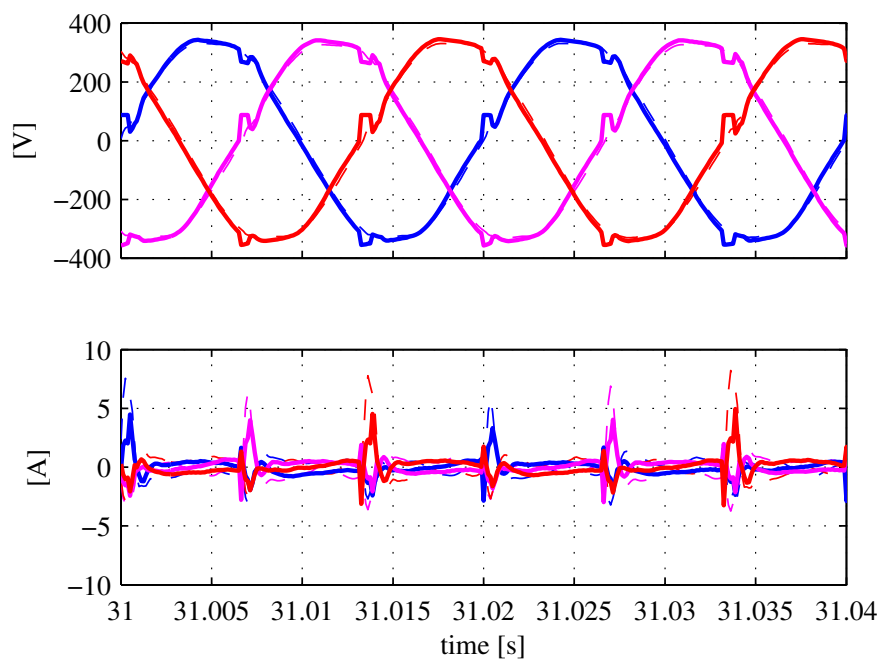

Fig. 11. Experimental results. a) LFSI and b) current response. Measured currents are shown in dashed lines and estimated in bold.
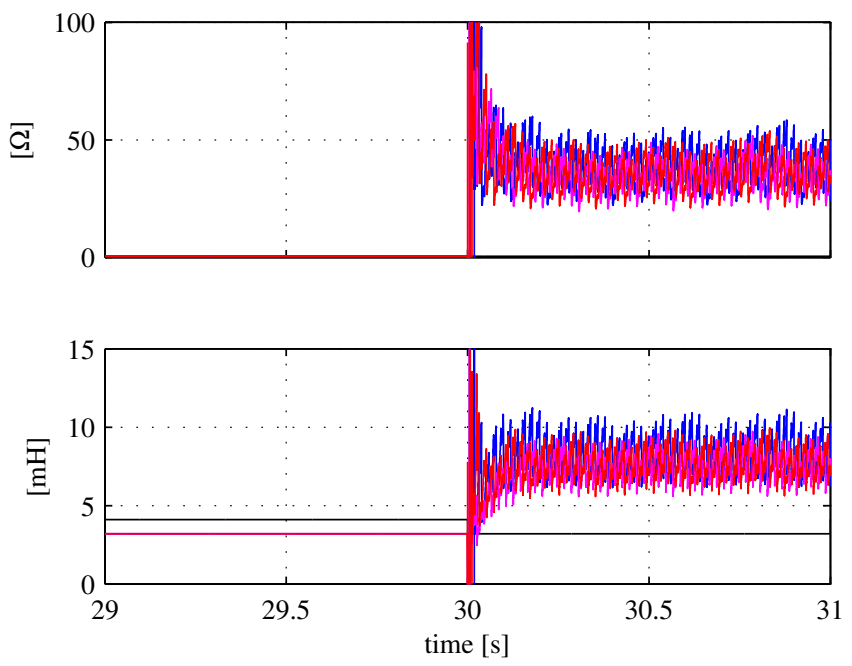

Fig. 12. Experimental results. Parameter estimation during a transient in the grid impedance. RLS estimation is enabled at $t=30 \mathrm{~s}$. a) Resistance estimation. b) Inductance estimation.

ditions. A good match between both components is obtained.

\section{CONCLUSION}

Estimation of the grid impedance is a key factor for improving the performance in weak networks. This paper has proposed the use of a LFSI technique to online estimate the impedance with a moderate computational burden for online implementation. The proposed system is triggered from the error signal coming from a Luenberger observer used for the control of the grid current in a LCL filter. The proposed observer and the estimation method have been tested through simulation and experimental results. Different methods for the LFSI have been compared and an the injection in the 


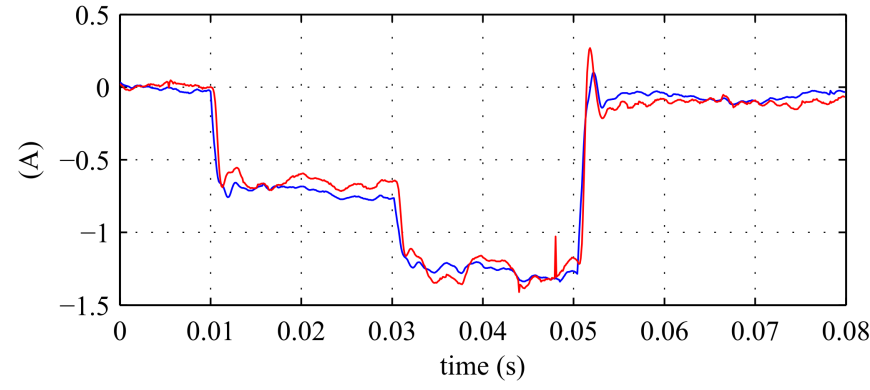

Fig. 13. Experimental results. Proposed observer working under different steps at the $i_{q}$ component. Active damping gain $K_{a}=10$.

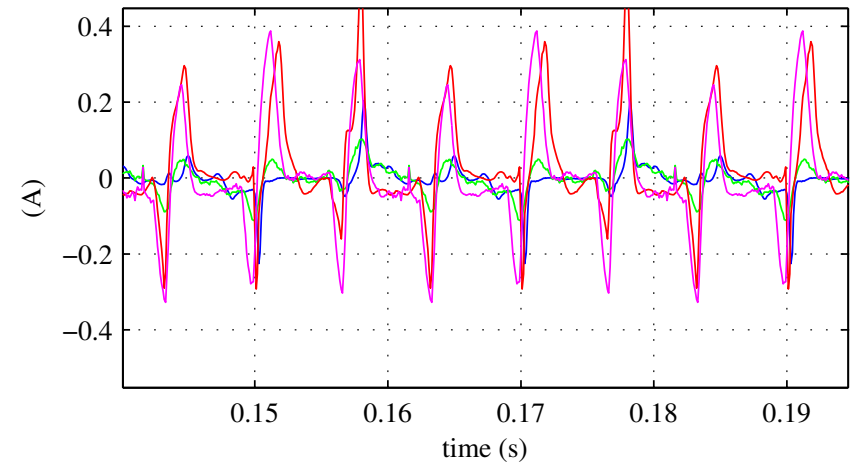

Fig. 14. Experimental results. Estimation of $i_{d}$ and $i_{q}$ currents using RLS at the synchronous reference frame and pulse injection based on Method\#3. The system was operated in close loop with both components having reference 0.

synchronous reference frame has been selected based on an increased sensitivity and reduced cross-coupling.

\section{REFERENCES}

[1] H. Xiao, X. Qu, S. Xie, and J. Xu, "Synthesis of active damping for grid-connected inverters with an LCL filter," in 2012 IEEE Energy Conversion Congress and Exposition (ECCE), Sep. 2012, pp. 550-556.

[2] J. Xu, S. Xie, and T. Tang, "Active Damping-Based Control for GridConnected -Filtered Inverter With Injected Grid Current Feedback Only," IEEE Transactions on Industrial Electronics, vol. 61, no. 9, pp. 47464758, Sep. 2014.

[3] W. Yao, M. Chen, J. Matas, J. Guerrero, and Z.-m. Qian, "Design and Analysis of the Droop Control Method for Parallel Inverters Considering the Impact of the Complex Impedance on the Power Sharing," IEEE Transactions on Industrial Electronics, vol. 58, no. 2, pp. 576-588, 2011.

[4] J. He, Y. W. Li, J. Guerrero, F. Blaabjerg, and J. Vasquez, "An Islanding Microgrid Power Sharing Approach Using Enhanced Virtual Impedance Control Scheme," IEEE Transactions on Power Electronics, vol. 28, no. 11 , pp. 5272-5282, 2013.

[5] M. Sumner, B. Palethorpe, and D. Thomas, "Impedance measurement for improved power quality-Part 2: a new technique for stand-alone active shunt filter control," IEEE Transactions on Power Delivery, vol. 19, no. 3, pp. 1457-1463, 2004.

[6] J. Massing, M. Stefanello, H. Grundling, and H. Pinheiro, "Adaptive Current Control for Grid-Connected Converters With LCL Filter," IEEE Transactions on Industrial Electronics, vol. 59, no. 12, pp. 4681-4693, 2012.

[7] M. Liserre, F. Blaabjerg, and R. Teodorescu, "Grid Impedance Estimation via Excitation of LCL -Filter Resonance," IEEE Transactions on Industry Applications, vol. 43, no. 5, pp. 1401-1407, 2007.

[8] D. Martin and E. Santi, "Auto tuning of digital deadbeat current controller for grid tied inverters using wide bandwidth impedance identification," in 2012 Twenty-Seventh Annual IEEE Applied Power Electronics Conference and Exposition (APEC), 2012, pp. 277-284.

[9] L. Asiminoaei, R. Teodorescu, F. Blaabjerg, and U. Borup, "A digital controlled PV-inverter with grid impedance estimation for ENS detection," IEEE Transactions on Power Electronics, vol. 20, no. 6, pp. 14801490, 2005.

[10] D. Reigosa, F. Briz, C. Charro, P. Garcia, and J. Guerrero, "Active Islanding Detection Using High-Frequency Signal Injection," IEEE Transactions on Industry Applications, vol. 48, no. 5, pp. 1588-1597, 2012.

[11] D. Reigosa, F. Briz, C. Blanco, P. Garcia, and J. Manuel Guerrero, "Active Islanding Detection for Multiple Parallel-Connected InverterBased Distributed Generators Using High-Frequency Signal Injection," IEEE Transactions on Power Electronics, vol. 29, no. 3, pp. 1192-1199, 2014.

[12] M. Sumner, B. Palethorpe, and D. Thomas, "Impedance measurement for improved power quality-Part 1: the measurement technique," IEEE Transactions on Power Delivery, vol. 19, no. 3, pp. 1442-1448, 2004.

[13] N. Hoffmann and F. Fuchs, "Minimal Invasive Equivalent Grid Impedance Estimation in Inductive \#x2013; Resistive Power Networks Using Extended Kalman Filter," IEEE Transactions on Power Electronics, vol. 29, no. 2, pp. 631-641, 2014.

[14] L. Asiminoaei, R. Teodorescu, F. Blaabjerg, and U. Borup, "Implementation and Test of an Online Embedded Grid Impedance Estimation Technique for PV Inverters," IEEE Transactions on Industrial Electronics, vol. 52, no. 4, pp. 1136-1144, 2005.

[15] A. Timbus, P. Rodriguez, R. Teodorescu, and M. Ciobotaru, "Line Impedance Estimation Using Active and Reactive Power Variations," in IEEE Power Electronics Specialists Conference, 2007. PESC 2007, 2007, pp. 1273-1279.

[16] F. Briz, A. Diez, and M. W. Degner, "Dynamic operation of carriersignal-injection-based sensorless direct field-oriented AC drives," IEEE Transactions on Industry Applications, vol. 36, no. 5, pp. 1360-1368, Oct. 2000.

[17] M. Liserre, F. Blaabjerg, and S. Hansen, "Design and control of an LCLfilter-based three-phase active rectifier," IEEE Transactions on Industry Applications, vol. 41, no. 5, pp. 1281-1291, Sep. 2005.

[18] D. Wojciechowski, "Novel predictive control of 3-phase LCL-based active power filter," in Compatibility and Power Electronics, 2009. CPE '09., May 2009, pp. 298-305.

[19] J. Dannehl, M. Liserre, and F. Fuchs, "Filter-Based Active Damping of Voltage Source Converters With Filter," IEEE Transactions on Industrial Electronics, vol. 58, no. 8, pp. 3623-3633, Aug. 2011.

[20] M. Liserre, A. Aquila, and F. Blaabjerg, "Genetic algorithm-based design of the active damping for an LCL-filter three-phase active rectifier," IEEE Transactions on Power Electronics, vol. 19, no. 1, pp. 76-86, Jan. 2004.

[21] S. Zhang, S. Jiang, X. Lu, B. Ge, and F. Z. Peng, "Resonance Issues and Damping Techniques for Grid-Connected Inverters With Long Transmission Cable," IEEE Transactions on Power Electronics, vol. 29, no. 1, pp. 110-120, Jan. 2014.

[22] R. Pena-Alzola, M. Liserre, F. Blaabjerg, R. Sebastian, J. Dannehl, and F. Fuchs, "Systematic Design of the Lead-Lag Network Method for Active Damping in LCL-Filter Based Three Phase Converters," IEEE Transactions on Industrial Informatics, vol. 10, no. 1, pp. 43-52, Feb. 2014.

[23] F. Huerta, E. Bueno, S. Cobreces, F. Rodriguez, and C. Giron, "Control of grid-connected voltage source converters with LCL filter using a Linear Quadratic servocontroller with state estimator," in IEEE Power Electronics Specialists Conference, 2008. PESC 2008, Jun. 2008, pp. 3794-3800.

[24] M. Malinowski and S. Bernet, "A Simple Voltage Sensorless Active Damping Scheme for Three-Phase PWM Converters With an Filter," IEEE Transactions on Industrial Electronics, vol. 55, no. 4, pp. 18761880, Apr. 2008.

[25] D. Luenberger, "Observing the State of a Linear System," IEEE Transactions on Military Electronics, vol. 8, no. 2, pp. 74-80, Apr. 1964.

[26] A. Arriagada, J. Espinoza, J. Rodriguez, and L. Moran, "On-line filtering reactance identification in voltage-source three-phase active-front-end rectifiers," in The 29th Annual Conference of the IEEE Industrial Electronics Society, 2003. IECON '03, vol. 1, Nov. 2003, pp. 192-197 vol.1. 